\title{
UPF1 Promotes Chemoresistance to Oxaliplatin Through Maintenance of Stemness by Increasing Phosphorylated TOP2A in Colorectal Cancer.
}

\section{Congcong Zhu}

Fudan University Shanghai Cancer Center

\section{Long Zhang}

Fudan University Shanghai Cancer Center

Senlin Zhao

Fudan University Shanghai Cancer Center

\section{Weixing Dai}

Fudan University Shanghai Cancer Center

\section{Yun Xu}

Fudan University Shanghai Cancer Center

\section{Yuqin Zhang}

Fudan University Shanghai Cancer Center

Hongtu Zheng

Fudan University Shanghai Cancer Center

\section{Weiqi Sheng}

Fudan University Shanghai Cancer Center

YeXu ( $\nabla$ yexu@shmu.edu.cn )

Fudan University Shanghai Cancer Center https://orcid.org/0000-0003-0455-4556

\section{Research}

Keywords: UPF1, Oxaliplatin, Chemoresistance, TOP2A, Colorectal cancer

Posted Date: December 22nd, 2020

DOl: https://doi.org/10.21203/rs.3.rs-130880/v1

License: (c) (i) This work is licensed under a Creative Commons Attribution 4.0 International License. Read Full License 


\section{Abstract}

Background: UPF1 is proved to dysregulate in multiple tumors and influence carcinogenesis. However, the role of UPF1 on oxaliplatin resistance in colorectal cancer (CRC) remains unknown.

Methods: Firstly, we investigated the clinical relevance of UPF1 in CRC patients. Then, we explored the influence of UPF1 on chemoresistance to oxaliplatin in vitro and in vivo. Finally, we disclosed the underlying mechanisms of oxaliplatin resistance induced by UPF1.

Results: UPF1 is upregulated in CRC and overexpression of UPF1 more likely results in recurrence in CRC patients and predicts a poorer overall survival (OS). UPF1 maintains stemness in CRC cell lines and promotes chemoresistance to oxaliplatin in CRC. UPF1-induced oxaliplatin resistance can be associated with interaction with TOP2A and increasing phosphorylated TOP2A.

Conclusions: UPF1 was overexpressed and predicted a poor prognosis in CRC. UPF1 enhanced the stemness and chemoresistance to oxaliplatin by interaction with TOP2A and increase of phosphorylated TOP2A in CRC, which may provide a new therapy strategy for chemoresistance to oxaliplatin in CRC patients.

\section{Introduction}

Colorectal cancer (CRC) is the third most frequent for incidence and the second most frequent for mortality worldwide [1]. While in China, CRC is the fourth most common and the fifth leading cause of cancer death [2]. Surgical resection is the potential radical treatment for CRC. Other than surgery, radiochemotherapy, targeted therapy and immunotherapy can be applied to CRC patients with lymph node metastases or distant metastases [3-5]. However, the acquisition of drug resistance is a major hurdle for good clinical prognosis [6].

Oxaliplatin, a third-generation platinum coordination complex, can be used for treatment in multiple cancers [7-9]. Oxaliplatin-based combined chemotherapy is routinely applied in advanced and metastatic $\mathrm{CRC}$ and significantly increases the overall survival rate and metastases resection rate [10]. Unfortunately, acquired or intrinsic resistance brings failure to treatment. The underlying mechanisms of oxaliplatin resistance include DNA damage response and repair, inhibition of cell death, cellular transport, detoxification and epigenetic alteration [11].

UPF1, an mRNA surveillance factor, is a RNA-dependent ATPase and helicase for nonsense-mediated decay (NMD) of mRNAs containing premature stop codons [12]. In addition, UPF1 is proved to dysregulate and influence carcinogenesis in multiple tumors. UPF1 is commonly mutated in pancreatic adenosquamous carcinoma (ASC) and there is little or no UPF1 expression in many ASC tumors compared to adjacent normal tissue [13]. It is revealed that UPF1 is downregulated in hepatocellular carcinoma and inhibits the tumor progression $[14,15]$. However, the role of UPF1 in oxaliplatin resistance in $\mathrm{CRC}$ remains unclear. 


\section{Materials And Methods}

\section{Patients and specimens}

76 patients were pathologically diagnosed with CRC and received surgical therapy at Fudan University Shanghai Cancer Center. Patients received neoadjuvant radiochemotherapy were excluded from our study. Specimens from the 76 patients were made into tissue microarrays (TMA). Our study was carried out in accordance with the requirements of the Biomedical Ethics Committee of Fudan University Shanghai Cancer Center.

\section{Cell lines and culture}

CRC cell lines (DLD1, HT29, LOVO, HCT116, RKO, SW480, SW620), normal colonic epithelial cell line (NCM460) and HEK-293T cell line were purchased from Type Culture Collection Cell Bank, Chinese Academy of Sciences. All cell lines were cultured in Dulbecco's Modified Eagles Medium (DMEM) with $10 \%$ fetal bovine serum (FBS), penicillin $\left(10^{7} \mathrm{U} / \mathrm{L}\right)$ and streptomycin $(10 \mathrm{mg} / \mathrm{L})$ and incubated at $37^{\circ} \mathrm{C}$ in a humidified atmosphere containing $5 \% \mathrm{CO}_{2}$ in Fudan University Shanghai Cancer Center.

\section{Lentivirus production and transfection}

Core plasmid was co-transfected with psPAX2 and pMD2.G into HEK-293T cells using Hieff Trans ${ }^{\text {TM }}$ Liposomal Transfection Reagent (Yeasen, Shanghai, China). 48 hours later, virus supernatant was collected. $3 \times 10^{5}$ cells were cultured in a 6-well plate. After incubation in virus supernatant for 48 hours, stable cell lines were selected with puromycin or flow cytometry and transfected efficiency was evaluated by immunoblot.

\section{Flow cytometry}

Apoptosis assay was conducted using Annexin V-PE/7-AAD apoptosis detection kit (Yeasen, Shanghai, China). Briefly, $3 \times 10^{5}$ cells were cultured in a 6 -well plate with or without being treated with oxaliplatin for 48 hours. Adherent cells and cells in supernatant were collected and washed by phosphate buffer saline (PBS) twice. Cells suspended with $1 \times$ binding buffer with the addition of $5 \mu \mathrm{L}$ Annexin $\mathrm{V}$ and $10 \mu \mathrm{L}$ 7-AAD. After incubation protecting from light for 15 minutes, apoptosis rate was detected by Flow cytometer.

\section{Western blotting}

Total protein was extracted with NuPAGE ${ }^{\circledR}$ LDS sample buffer (Thermo Fisher Scientific, Waltham, USA). A certain amount of protein was separated by SDS-PAGE gel and transferred to PVDF membranes. After blockage with non-fat milk powder and incubation with primary antibodies and horseradish peroxidaseconjugated secondary antibodies, images were captured with ImageQuant ${ }^{\text {tM }}$ biomolecular imager. All the antibodies used in our study were listed in additional file named Table S1.

\section{Drug cytotoxicity assay}


Drug cytotoxicity was evaluated by Cell Counting Kit-8 (CCK-8) assay. Cell suspension was seeded in 96well plate and cells were treated with a gradient concentration of oxaliplatin for 48 hours after adhesion. Medium containing CCK-8 reagent (Yeasen, Shanghai, China) was added to each well of the plate. After two-hours' incubation, absorbance at $450 \mathrm{~nm}$ was detected by microplate reader.

\section{Clone formation assay}

1000 cells were cultured in a 6-well plate and treated with oxaliplatin for 48 hours after adhesion. The medium was replaced by the fresh every three days. 2 weeks later, cells were fixed with $4 \%$ paraformaldehyde for 30 minutes and stained with crystal violet for 30 minutes. Images were photographed and visible colonies were counted.

\section{Co-immunoprecipitation (Co-IP) assays and mass spectrometry}

Cells in culture dish were lysed with IP buffer containing protease inhibitors and phosphatase inhibitor cocktail (Topscience, Shanghai, China). IP buffer was a mixture of 1 mM EDTA, 20 mM HEPES, $150 \mathrm{mM}$ $\mathrm{NaCl}, 0.05 \%$ sodium deoxycholate and $0.05 \% \mathrm{NP}-40$. Lysate was spun at the speed of $12,000 \mathrm{rpm}$ at $4{ }^{\circ} \mathrm{C}$ for 12 minutes. $60 \mu \mathrm{L}$ was kept for input and the rest of the extract was immunoprecipitated with anti-HA beads or anti-FLAG resins at $4{ }^{\circ} \mathrm{C}$ for more than 3 hours. After washing 5 times with IP buffer, beads or resins of protein bound were lysed with $1.25 \times$ SDS loading buffer and boiled at $100{ }^{\circ} \mathrm{C}$ for 10 minutes. Bands appeared after electrophoresis and silver staining in SDS-PAGE gel received mass spectrometry analysis (Wayen Biotechnologies, Shanghai, China) for protein identification. Interacted protein was further confirmed by immunoblot.

\section{Mammosphere formation assay}

Mammosphere formation assay was conducted as previously described [16]. Cells were digested by trypsin and washed by PBS twice. 300 cells suspended in $200 \mu \mathrm{L}$ mammosphere medium were seeded in 96-well plate with ultra-low attachment surface. The formulation of mammosphere medium was shown in additional file named Table S2. 10 days later, images were photographed and mammosphere were counted.

\section{Immunohistochemistry (IHC)}

IHC was performed as previously described [17]. The results of IHC staining were determined by immunoreactive score (IRS), ranging from 0 to 12. IRS equals the number of positive cells multiplied by the staining intensity. The number of cells stained were divided into 5 groups: $0,1-10 \%, 11-50 \%, 51-80 \%$ and $81-100 \%$, corresponding to 0 - 4 score respectively. And staining intensity has 4 levels, negative, weak, moderate and strong, corresponding to 0 - 3 score respectively.

\section{Immunocytochemistry (ICC)}


Sterilized round coverslips were laid in a 24-well plate and cells were seeded on them. After 24 hours incubation, cells were fixed with $4 \%$ paraformaldehyde for 30 minutes and permeabilized on ice with $0.25 \%$ Triton X-100. Then cells were blocked with BSA and incubated with primary antibodies for 2 hours and fluorescent secondary antibodies protecting from light for 1 hour at room temperature. After mounting with DAPI Fluoromount mounting medium for 15 minutes, images were captured using Leica confocal system.

\section{Xenograft experiments}

$5 \times 10^{6}$ cells were subcutaneously injected into four-week-old male BALB/c nude mice. 5 days later, mice were randomly divided into groups and treated with oxaliplatin $(5 \mathrm{mg} / \mathrm{kg})$ or $5 \%$ glucose solution by intraperitoneal injection, twice per week for three weeks, respectively. All mice were sacrificed and tumors were collected and weighed. Tumor volume equaled length $\times$ width^$^{\wedge} 2 \times 0.5$ and was measured twice a week. Xenografts were saved in $4 \%$ paraformaldehyde for following experiments. Tumor weight and volume were presented as means \pm standard deviation (SD). Apoptosis rates in the xenograft were determined by terminal deoxynucleotidyl transferase mediated dUTP nick end labeling (TUNEL) technology. Xenograft experiments were approved by the Committee on Animals Handling of Fudan University Shanghai Cancer Center.

\section{Statistical analysis}

Data are shown in mean \pm SD. All analyses were performed by IBM SPSS 22.0 software. Quantitative variables were analyzed using Student's t-test. The Log-rank test in the Kaplan-Meier method and the Cox regression model were used to assess patients' survival outcome and prognostic factors. All the experiments were performed in triplicate. A two-tailed value of $P<0.05$ was considered statistically

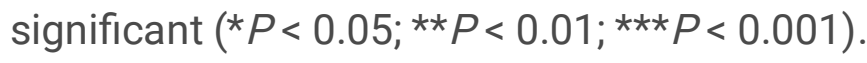

\section{Results}

\section{UPF1 is upregulated and predicts a poor prognosis in CRC.}

To investigate the expression of UPF1 in CRC tissues, we performed bioinformatics analysis of UPF1 in mRNA level using the public RNA sequencing (RNA-seq) datasets from The Cancer Genome Atlas (TCGA) and found that UPF1 was overexpressed ( $P=0.043$, Fig. 1a). we also detected it by IHC staining of TMA using a retrospective cohort containing 76 patients with paired CRC tumor and normal tissues. Similarly, the results verified that UPF1 was dramatically upregulated in CRC tissues $(P<0.001, \mathrm{Fig}$. 1c). The representative IHC images of UPF1 staining were shown in Fig. 1b. In these patients, UPF1-positive was detected in $52(68.4 \%)$ of the tumor tissues, whereas only 4 (5.3\%) of the normal tissues was UPF1positive (Table $1, P<0.001$ ). 
Table 1

Clinicopathological characteristics of CRC patients in according to Upf1 or recurrence status

$\begin{array}{lllllll}\begin{array}{l}\text { All } \\ \text { cases }\end{array} & \begin{array}{l}\text { Upf1 } \\ \text { negative }\end{array} & \begin{array}{l}\text { Upf1 } \\ \text { positive }\end{array} & \begin{array}{l}P \\ \text { value }\end{array} & \begin{array}{l}\text { Non- } \\ \text { recurrence }\end{array} & \text { recurrence } & \begin{array}{l}P \\ \text { value }\end{array} \\ (n=76) & & & & & & \end{array}$

Tissue

0.000

\begin{tabular}{llll} 
Normal & 76 & 72 & 4 \\
\hline Tumor & 76 & 24 & 52 \\
\hline
\end{tabular}

Age

$<60$

$45 \quad 14$

(59.2\%)

$14 \quad 31$

$\geq 60$

$\begin{array}{lll}31 & 10 & 21\end{array}$

$\begin{array}{lll}(40.8 \%) & (41.7 \%) \quad(40.4 \%)\end{array}$

0.916

0.380

Gender

Female

35

$(46.1 \%)$

12

$(50.0 \%)$

23

(44.2\%)

$\begin{array}{ll}26(55.3 \%) & 19 \\ & (65.5 \%)\end{array}$

(59.6\%)

$21(44.7 \%) \quad 10$

(34.5\%)

Male

41

(53.9\%)

12

(50.0\%)

29

(55.8\%)

0.639

0.265

Tumor size

$<4 \mathrm{~cm}$

41

(53.9\%)

10

(41.7\%)

(59.6\%)

$24(51.1 \%)$

11

(37.9\%)

$\geq 4 \mathrm{~cm}$

$\begin{array}{lll}35 & 14 & 21 \\ (46.1 \%) & (58.3 \%) & (40.4 \%)\end{array}$

0.145

$23(48.9 \%) \quad 18$

(62.1\%)

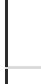

Tumor location

Left-sided

29

(38.2\%)

$9(37.5 \%)$

20

(38.5\%)

0.444

0.299

Right-sided

$\begin{array}{lll}25 & 10 & 15 \\ (32.9 \%) & (41.7 \%) & (28.8 \%)\end{array}$

Rectum

$22.9 \%)$

$5(20.8 \%)$

17
$(32.7 \%)$

Differentiation

Well/moderate

\begin{tabular}{lll}
$\begin{array}{l}64 \\
(84.2 \%)\end{array}$ & $\begin{array}{l}17 \\
(70.8 \%)\end{array}$ & $\begin{array}{l}47 \\
(90.4 \%)\end{array}$ \\
\hline 12 & $7(29.2 \%)$ & $5(9.6 \%)$ \\
$(15.8 \%)$ & & \\
\hline
\end{tabular}

0.067

$\begin{array}{ll}15(31.9 \%) & 14 \\ & (48.3 \%)\end{array}$

$\begin{array}{ll}26(55.3 \%) & 15 \\ & (51.7 \%)\end{array}$

$21(44.7 \%) \quad 14$

(48.3\%)

0.760

Poor

$P$ value in bold and italic indicates significant. 


\begin{tabular}{|c|c|c|c|c|c|c|c|}
\hline & $\begin{array}{l}\text { All } \\
\text { cases } \\
(n=76)\end{array}$ & $\begin{array}{l}\text { Upf1 } \\
\text { negative }\end{array}$ & $\begin{array}{l}\text { Upf1 } \\
\text { positive }\end{array}$ & $\begin{array}{l}P \\
\text { value }\end{array}$ & $\begin{array}{l}\text { Non- } \\
\text { recurrence }\end{array}$ & recurrence & $\begin{array}{l}P \\
\text { value }\end{array}$ \\
\hline $\begin{array}{l}\text { Lymphovascular } \\
\text { invasion }\end{array}$ & & & & 0.983 & & & 1.000 \\
\hline Negative & $\begin{array}{l}68 \\
(89.5 \%)\end{array}$ & $\begin{array}{l}22 \\
(91.7 \%)\end{array}$ & $\begin{array}{l}46 \\
(88.5 \%)\end{array}$ & & 42 (89.2\%) & $\begin{array}{l}26 \\
(89.7 \%)\end{array}$ & \\
\hline Positive & $\begin{array}{l}8 \\
(10.5 \%)\end{array}$ & $2(8.3 \%)$ & $\begin{array}{l}6 \\
(11.5 \%)\end{array}$ & & $5(10.6 \%)$ & 3 (10.3\%) & \\
\hline $\begin{array}{l}\text { Perineural } \\
\text { invasion }\end{array}$ & & & & 0.631 & & & 0.178 \\
\hline Negative & $\begin{array}{l}64 \\
(84.2 \%)\end{array}$ & $\begin{array}{l}19 \\
(79.2 \%)\end{array}$ & $\begin{array}{l}45 \\
(86.5 \%)\end{array}$ & & 37 (78.7\%) & $\begin{array}{l}27 \\
(93.1 \%)\end{array}$ & \\
\hline Positive & $\begin{array}{l}12 \\
(15.8 \%)\end{array}$ & $5(20.8 \%)$ & $\begin{array}{l}7 \\
(13.5 \%)\end{array}$ & & $10(21.3 \%)$ & $2(6.9 \%)$ & \\
\hline TNM stage & & & & 0.625 & & & 0.040 \\
\hline Q & $\begin{array}{l}7 \\
(9.2 \%)\end{array}$ & $2(8.3 \%)$ & $5(9.6 \%)$ & & $6(12.8 \%)$ & $1(3.4 \%)$ & \\
\hline प & $\begin{array}{l}35 \\
(46.1 \%)\end{array}$ & $\begin{array}{l}13 \\
(54.2 \%)\end{array}$ & $\begin{array}{l}22 \\
(42.3 \%)\end{array}$ & & $25(53.2 \%)$ & $\begin{array}{l}10 \\
(34.5 \%)\end{array}$ & \\
\hline$\square$ & $\begin{array}{l}34 \\
(44.7 \%)\end{array}$ & 9 (37.5\%) & $\begin{array}{l}25 \\
(48.1 \%)\end{array}$ & & $16(34.0 \%)$ & $\begin{array}{l}18 \\
(62.1 \%)\end{array}$ & \\
\hline CEA level & & & & 0.692 & & & 0.361 \\
\hline$<5 \mathrm{ng} / \mathrm{mL}$ & $\begin{array}{l}53 \\
(69.7 \%)\end{array}$ & $\begin{array}{l}16 \\
(66.7 \%)\end{array}$ & $\begin{array}{l}37 \\
(71.2 \%)\end{array}$ & & 31 (66.0\%) & $\begin{array}{l}22 \\
(75.9 \%)\end{array}$ & \\
\hline$\geq 5 \mathrm{ng} / \mathrm{mL}$ & $\begin{array}{l}23 \\
(30.3 \%)\end{array}$ & 8 (33.3\%) & $\begin{array}{l}15 \\
(28.8 \%)\end{array}$ & & $16(34.0 \%)$ & 7 (24.1\%) & \\
\hline $\begin{array}{l}\text { TOP2A } \\
\text { expression }\end{array}$ & & & & 0.012 & & & 0.530 \\
\hline Negative & $\begin{array}{l}18 \\
(23.7 \%)\end{array}$ & $\begin{array}{l}10 \\
(41.7 \%)\end{array}$ & $\begin{array}{l}8 \\
(15.4 \%)\end{array}$ & & $10(21.3 \%)$ & $8(27.6 \%)$ & \\
\hline Positive & $\begin{array}{l}58 \\
(76.3 \%)\end{array}$ & $\begin{array}{l}14 \\
(58.3 \%)\end{array}$ & $\begin{array}{l}44 \\
(84.6 \%)\end{array}$ & & 37 (78.7\%) & $\begin{array}{l}21 \\
(72.4 \%)\end{array}$ & \\
\hline Upf1 expression & & & & & & & 0.035 \\
\hline Negative & $\begin{array}{l}24 \\
(31.6 \%)\end{array}$ & & & & 19 (40.4\%) & $5(17.2 \%)$ & \\
\hline
\end{tabular}

$P$ value in bold and italic indicates significant. 


\begin{tabular}{|c|c|c|c|c|c|c|c|}
\hline & $\begin{array}{l}\text { All } \\
\text { cases } \\
(n=76)\end{array}$ & $\begin{array}{l}\text { Upf1 } \\
\text { negative }\end{array}$ & $\begin{array}{l}\text { Upf1 } \\
\text { positive }\end{array}$ & $\begin{array}{l}P \\
\text { value }\end{array}$ & $\begin{array}{l}\text { Non- } \\
\text { recurrence }\end{array}$ & recurrence & $\begin{array}{l}P \\
\text { value }\end{array}$ \\
\hline Positive & $\begin{array}{l}52 \\
(68.4 \%)\end{array}$ & & & & $28(59.6 \%)$ & $\begin{array}{l}24 \\
(82.8 \%)\end{array}$ & \\
\hline
\end{tabular}

To explore the clinical significance of UPF1 in CRC, we analyzed the correlation between the expression of UPF1 and clinicopathological characteristics of CRC patients (Table 1). A significant association was observed between UPF1 positive and TOP2A positive $(P=0.012,58.3 \%$ in UPF1-negative group vs $84.6 \%$ in UPF1-positive group). High UPF1 expression $(P=0.035)$ and high TNM stage $(P=0.040)$ more likely result in recurrence in CRC patients. However, high expression of UPF1 and recurrence in CRC patients had no correlation with gender, age, tumor location, tumor size, lymphovascular invasion, perineural invasion and carcinoembryonic antigen (CEA) level. Additionally, Kaplan-Meier curves showed a strong correlation between UPF1 higher expression and a poorer overall survival (OS) ( $P=0.045$, Fig. 1d). UPF1positive patients also had a shorter recurrence-free survival (RFS) $(P=0.029$, Fig. 1e). In addition, univariate and multivariate $\mathrm{COX}$ regression analysis suggested that UPF1-positive $(\mathrm{HR}=3.719 ; 95 \% \mathrm{Cl}=$ 1.313 to $10.529 ; P=0.013)$ and poor differentiation $(\mathrm{HR}=2.927 ; 95 \% \mathrm{Cl}=1.186$ to $7.222 ; P=0.020)$ were independent prognostic risk factors for RFS in CRC (Fig. 1f, $1 \mathrm{~g}$ ). In summary, UPF1 is aberrantly upregulated in CRC and high expression of UPF1 predicts a poor prognosis in CRC patients.

\section{UPF1 promotes oxaliplatin resistance in CRC in vitro.}

The baseline expression of UPF1 in CRC cell lines and normal colonic epithelial cell line was detected by immunoblot. Notably, UPF1 expression in NCM460 was remarkably less compared with CRC cell lines (Fig. 2a). We chose DLD1 to overexpress UPF1 due to its relative low baseline expression. And based on the same rule, UPF1 was knocked down in HCT116 using three small hairpin RNAs. Overexpression and knockdown were confirmed by western blotting and $\mathrm{SH} 2$ was the most efficient in UPF1 knockdown (Fig. 2b, 2c). HA and FLAG tag were used for co-immunoprecipitation (Co-IP) assay. Drug cytotoxicity assay showed that overexpression of UPF1 decreased oxaliplatin sensitivity in DLD1 while knockdown of UPF1 increased oxaliplatin sensitivity in HCT116 (Fig. 2d, 2e). In apoptosis assay, overexpression and knockdown of UPF1 had no influence on apoptosis rate in DLD1 and HCT116 respectively. Whereas after treatment for 48 hours, oxaliplatin-induced apoptosis was significantly reduced in DLD1-UPF1 and raised in HCT116-shUPF1 (Fig. 2f-2i). Clone formation assay also affirmed that UPF1 promoted oxaliplatin resistance in CRC in vitro. The number of colonies were more in DLD1-UPF1 group and less in HCT116shUPF1 group compared with control group with treatment of oxaliplatin for 48 hours (Fig. 2j, 2 k). However, the size of colonies remained no obvious change between different groups, which indicated that UPF1 may have no effect on cell proliferation. CCK-8 assay also showed that UPF1 did not influence proliferation in DLD1 and HCT116 cell lines (Fig. S1d, S1e). 


\section{UPF1 promotes oxaliplatin resistance in CRC in vivo}

Nude mice were divided into 2 groups and injected with HCT116-shNC and HCT116-shUPF1 cell lines respectively. One half of each group was treated with $5 \%$ glucose solution (GS) and the other was treated with Oxaliplatin $(5 \mathrm{mg} / \mathrm{kg})$. The tumor xenografts were shown in Fig. 3a. In the groups treated with GS, there is no significant difference between HCT116-shNC and HCT116-shUPF1 groups. Whereas, xenografts in HCT116-shNC group grew faster and were much larger and heavier after treatment with oxaliplatin (Fig. 3b, 3c). In line, these findings were further confirmed by TUNEL assay (Fig. 3d). Apoptosis rate in tissues remained no change in HCT116-shUPF1 but after being treated with oxaliplatin, apoptosis rate remarkably raised compared with control. xenografts assay in vivo revealed that UPF1 promotes oxaliplatin resistance in CRC.

\section{UPF1 interacts with TOP2A and increases the level of phosphorylated TOP2A.}

The protein in DLD1-UPF1 and DLD1-Ctrl cell lines with FLAG tag was lysed by IP buffer. After immunoprecipitation with anti-FLAG resin, protein extracts were separated in 10\% SDS-PAGE gel. some signature bands of DLD1-UPF1 cell line emerged after silver staining (Fig. 4a). SDS-PAGE gel containing signature bands was used for mass spectrometry. 167 kind of proteins were identified which were listed in additional file named Table S3. TOP2A, TOP1, CCAR2 and XRCC6 were selected as candidate proteins interacted with UPF1 according to pathway analysis (Fig. S1f) and biological function. The interaction between UPF1 and TOP2A was proved in co-IP assay. The results showed co-IP of TOP2A with UPF1FLAG using an anti-FLAG antibody. Similarly, UPF1 was also precipitated using an anti-HA antibody in HEK-293T cell line transfected into TOP2A-HA (Fig. 4b, 4c). In DLD1 cell line transfected into UPF1-FLAG, the results of ICC also indicated the colocalization of UPF1 and TOP2A (Fig. 4e). We further found that expression of total TOP2A remained no significant change but phosphorylated TOP2A at the site of Ser ${ }^{1106}$ was increased after upregulation of UPF1. Correspondingly, silencing UPF1 had no influence on total TOP2A expression but attenuated phosphorylated TOP2A at the same site (Fig. 4d).

To identify the binding region between UPF1 and TOP2A, we generated the truncated mutants of UPF1 and TOP2A to perform co-IP assays. The full length of TOP2A contains 1531 amino acids and six truncated mutants were generated (Fig. 5a, 5b). Co-IP assay revealed that Toprim domain may be the binding region with UPF1 (Fig. 5c). The full length of UPF1 contains 1129 amino acids and five truncated mutants were generated (Fig. 5d, 5e). The results demonstrated that the zinc finger domain of UPF1 may interact with TOP2A (Fig. 5f).

To verify the function of TOP2A in UPF1-induced oxaliplatin resistance, TOP2A was silenced using three small hairpin RNAs. Sh1 was selected for following experiments due to its biggest efficiency (Fig. 6a). Of note, drug cytotoxicity assay demonstrated that UPF1-induced resistant cells regained the sensitivity to oxaliplatin after knockdown of TOP2A (Fig. 6c). As shown in clone formation assay, DLD1-Ctrl, DLD1UPF1 and DLD1-UPF1-shTOP2A were treated by oxaliplatin for 48 hours. We can see that UPF1 overexpression significantly increase the number of colonies while the effect was abolished by silencing 
the TOP2A gene in the cells. The size of colonies had no difference among different groups (Fig. 6d, 6e). The results in apoptosis assay came to the same conclusion (Fig. $6 \mathrm{f}, 6 \mathrm{~g}$ ). In vivo, xenografts in DLD1UPF1 group were much larger and heavier under treatment with oxaliplatin. The trend of chemoresistance was brought to a halt with TOP2A knockdown (Fig. 6h). In same, TUNEL assay testified that inhibition of apoptosis in tissue induced by UPF1 was deactivated by silencing TOP2A (Fig. 6i). Altogether, these data strongly suggested that TOP2A played an essential role in the UPF1-induced chemoresistance to oxaliplatin in CRC.

\section{UPF1 maintains stemness of CRC cell lines.}

Cancer stem cells (CSCs) are considered as a subpopulation of cells in the tumor characterized by an increased ability to self-renew and seed secondary tumors [18]. The ability was defined as CSC-like properties or stemness. Studies have identified an essential role of CSCs in the drug resistance in colorectal cancer progression $[19,20]$. Mammosphere formation assay can be used to define the functional CSC-like properties in vitro and larger size and bigger number of mammosphere indicate enhanced stemness. In our study, the ability of mammosphere formation was enhanced after upregulation of UPF1 in DLD1 and was attenuated after knockdown of UPF1 in HCT116 (Fig. 7a, 7b).

Notably, UPF1-induced CSC-like properties was abated by silencing the expression of TOP2A (Fig. 7e, 7f).

EpCAM has been proposed as one of the CSC markers [21-23]. In our study, the percentage of EpCAMpositive cells was raised in DLD1-UPF1 and diminished in HCT116-shUPF1 (Fig. 7c, 7d). UPF1-induced change of EpCAM + cell rates was abolished by knockdown of TOP2A (Fig. 7g, 7 h).

\section{Discussion}

CRC maintains a high rate of incidence and mortality worldwide, as well as that in China $[1,2]$. For CRC

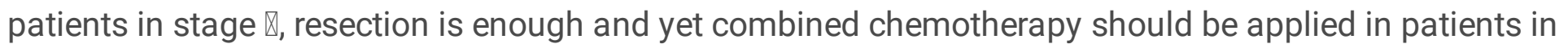
high-risk stage $\nabla$ and stage $\nabla / \nabla$. Stage $\nabla C R C$ exhibits a recurrence rate of about $20 \%$ after radical resection [24]. For patients with stage $₫$ disease, recurrence rate can be as high as more than $50 \%$ [25]. Chemotherapy resistance is a major cause of recurrence and poor prognosis in CRC patients [26].

Oxaliplatin, irinotecan and fluoropyrimidine are three backbones of first-line systemic treatments for CRC. As a single agent, oxaliplatin has limited efficacy applied as treatment for CRC patients [27]. Combination of oxaliplatin and fluorouracil is promising in treatment for the disease due to its highly synergistic effect [28]. Oxaliplatin-based combined chemotherapy is routinely applied in advanced and metastatic CRC and significantly increases the overall survival rate and metastases resection rate [10]. Unfortunately, drug resistance brings failure to treatment, which becomes a pressing problem in clinical treatment in CRC patients.

In our study, we used wild type CRC cell line HCT116 (HCT116-wt) to select over times with a gradual increasing concentration of oxaliplatin for acquired oxaliplatin-resistant cell line (HCT116-L). RNA-seq (Genminix Informatics, Shanghai, China) was used for transcriptome-wide analysis of differential gene 
expression in HCT116-wt and HCT116-L cell lines (Fig. S2). And we found that UPF1 was highly expressed in HCT116-L cell line.

It is reported that UPF1 can play a role in regulated mRNA and protein decay. Feng Q et al. reported that UPF1 acted as an E3 ubiquitin ligase to repress human skeletal muscle differentiation [29]. In addition, UPF1 is proved to dysregulate in multiple tumors and influence carcinogenesis [13-15]. However, there is lack of detailed study of UPF1 in CRC. It is reported that UPF1-mediated NMD might play a part in the selection of target gene mutations with a functional role in MSI-H carcinogenesis [30]. In line, Ada Collura et al. ascertained that inhibition of NMD in vivo using amlexanox reduced MSI tumor growth [31]. Currently, the function of UPF1 and potential mechanisms remains unclear in CRC.

Firstly, we demonstrated that UPF1 was aberrantly overexpressed in CRC tissues in mRNA and protein levels by bioinformatics analysis and IHC staining respectively. And high UPF1 expression more likely resulted in recurrence and predicted a worse OS in CRC patients. In addition, COX regression analysis suggested that UPF1-positive ( $\mathrm{HR}=3.719 ; 95 \% \mathrm{Cl}=1.313$ to $10.529 ; P=0.013)$ was an independent prognostic risk factor for RFS in CRC.

Herein, how UPF1 acts as a oncogene in CRC needed deep study. CCK-8, clone formation assays and flow cytometry indicated that UPF1 may have no effect on cell proliferation and apoptosis. While assays in vitro showed that overexpression of UPF1 weakened sensitivity to oxaliplatin in DLD1 while knockdown of UPF1 reinforced sensitivity to oxaliplatin in HCT116. In vivo, we reached the same conclusion that UPF1 promotes oxaliplatin resistance in CRC.

Cancer stem cells (CSCs) are defined by their functional properties and could be able to self-renew and propagate the tumor [32]. CSC-like properties or stemness have an essential role in the drug resistance in CRC $[19,20]$. Mammosphere formation assay can be used to define the functional stemness in vitro [16]. In our study, the stemness was progressed in UPF1-upregulation cell line and was impeded in UPF1knockdown cell line. CSCs are characterized by specific markers, such as EpCAM. Flow cytometry indicated that the percentage of EpCAM-positive cells was diminished after silencing UPF1 and increased after upregulating UPF1.

Afterwards, the underlying mechanism of UPF1-induced oxaliplatin resistance remained to be revealed. Mass spectrometry analysis identified 166 kind of proteins that may interact with UPF1. Enrichment analysis exhibited related pathways and TOP2A was unearthed to be involved in platinum-resistant pathway. TOP2A, DNA topoisomerase, an enzyme that controls and alters the topologic states of DNA during transcription. Phosphorylation of serine 1106 in the catalytic domain of TOP2A regulates enzymatic activity and drug sensitivity [33,34]. TOP2A is upregulated and could induce tumor development and progression in multiple tumors [35-38] and is proved important therapeutic targets of anticancer agents [39]. Etoposide, as an inhibitor of TOP2A, is a kind of cell cycle specific antitumor drug and applied in multiple tumors. In CRC tissues, TOP2A was proved to be upregulated in mRNA and protein levels (Fig. S1a-S1C). In the IHC staining of TMA, a significant association was observed between UPF1 positive and TOP2A positive. TOP1 could modulate colorectal cancer response to irinotecan [40]. 
CCAR2 overexpression decreased the chemosensitivity to oxaliplatin in CRC [41]. XRCC6 as a DNA repair gene may participate in platinum resistance by modulating the DNA repair capacity [42]. TOP2A, TOP1, CCAR2 and XRCC6 were selected as candidates according to pathway analysis and biological function. Forward and reverse co-IP assay proved the interaction of UPF1 with TOP2A, instead of TOP1, CCAR2 and XRCC6. In DLD1 transfected into UPF1-FLAG, the results of ICC assays also indicated the colocalization of UPF1 and TOP2A. To identify the binding region between UPF1 and TOP2A, we generated the truncated mutants of UPF1 and TOP2A to perform co-IP assays. Toprim in TOP2A is a catalytic domain involved in DNA strand breakage and rejoining and may be the binding region with UPF1. The zinc fingers vary widely in structure, as well as in function. And this domain of UPF1 may interact with TOP2A. We further found that expression of total TOP2A remained no significant change after upregulation of UPF1. While the interaction with UPF1 increased phosphorylated TOP2A at the site of Ser ${ }^{1106}$ thus may enhance the enzyme activity of TOP2A. Of note, UPF1-induced resistant cells regained the sensitivity to oxaliplatin after knockdown of TOP2A. We can see that UPF1 overexpression significantly reduced cytotoxicity induced by oxaliplatin while the resistance was abolished by silencing the TOP2A gene in the cells. Notably, UPF1-induced CSC-like properties was abated by silencing the expression of TOP2A. Altogether, these data strongly suggested that TOP2A played an essential role in the UPF1-induced chemoresistance of CRC cells in response to oxaliplatin in vitro and in vivo and TOP2A could be a therapeutic target in UPF1-overexpressed CRC patients.

There are some limitations in this study that could be addressed in future research. We have not figured out how interaction between UPF1 and TOP2A increases the level of phosphorylated TOP2A. And we wonder whether combination with the drugs targeted for TOP2A could make synergistic effect in patients with highly expressed UPF1.

\section{Conclusions}

UPF1 was overexpressed and predicted a poor prognosis in CRC. UPF1 promoted the stemness and chemoresistance to oxaliplatin by interaction with TOP2A and increasing phosphorylated TOP2A, which may provide a new therapy strategy for chemoresistance to oxaliplatin in CRC patients.

\section{Abbreviations}

CRC: colorectal cancer; OS: overall survival; NMD: nonsense-mediated decay; ASC: adenosquamous carcinoma; TMA: tissue microarrays; CCK-8: Cell Counting Kit-8; IP: immunoprecipitation; IHC: immunohistochemistry; IRS: immunoreactive score; ICC: immunocytochemistry; TUNEL: terminal deoxynucleotidyl transferase mediated dUTP nick end labeling; SD: standard deviation; TCGA: The Cancer Genome Atlas; CEA: carcinoembryonic antigen; RFS: recurrence-free survival; CSC: cancer stem cell; GS: glucose solution.

\section{Declarations}




\section{Ethics approval and consent to participate}

Our study was carried out in accordance with the requirements of the Biomedical Ethics Committee of Fudan University Shanghai Cancer Center. Informed consent was obtained from all patients included in the study. Xenograft experiments was approved by the Committee on Animals Handling of Fudan University Shanghai Cancer Center.

\section{Consent for publication}

Not applicable

\section{Availability of data and materials}

The data used to support the findings of this study are included within the article and the supplementary materials.

\section{Competing interests}

The authors declare that they have no competing interests.

\section{Funding}

The financial support of our study was from the Science and Technology Commission of Shanghai Municipality (19511121202, 20DZ1100100).

\section{Authors' contributions}

Ye Xu and WQ Sheng conceived the experiments. CC Zhu and L Zhang conducted the experiments. CC Zhu, L Zhang and SL Zhao analyzed and interpreted the data. WX Dai contributed to analyze the data. CC Zhu, Yun Xu and YQ Zhang prepared the figures. CC Zhu wrote the first draft and HT Zheng revised the manuscript. All authors read and approved the final manuscript.

\section{Acknowledgements}

I would like to extend my deep gratitude to Pro. Ming Yao and Pro. Xianghuo He for their guidance and support for our study.

\section{References}

1. Bray F, Ferlay J, Soerjomataram I, et al. Global cancer statistics 2018: GLOBOCAN estimates of incidence and mortality worldwide for 36 cancers in 185 countries. CA Cancer $\mathrm{J}$ Clin. 2018;68(6):394-424.

2. Chen W, Sun K, Zheng R, et al. Cancer incidence and mortality in China, 2014. Chin J Cancer Res. 2018;30(1):1-12. 
3. Ganesh K, Stadler ZK, Cercek A, et al. Immunotherapy in colorectal cancer: rationale, challenges and potential. Nat Rev Gastroenterol Hepatol. 2019;16(6):361-75.

4. Rosello $S$, Papaccio F, Roda $D$, et al. The role of chemotherapy in localized and locally advanced rectal cancer: A systematic revision. Cancer Treat Rev. 2018;63:156-71.

5. Zhai Z, Yu X, Yang B, et al. Colorectal cancer heterogeneity and targeted therapy: Clinical implications, challenges and solutions for treatment resistance. Semin Cell Dev Biol. 2017;64:10715.

6. Hammond WA, Swaika A, Mody K. Pharmacologic resistance in colorectal cancer: a review. Ther Adv Med Oncol. 2016;8(1):57-84.

7. Edeline J, Benabdelghani M, Bertaut A, et al. Gemcitabine and Oxaliplatin Chemotherapy or Surveillance in Resected Biliary Tract Cancer (PRODIGE 12-ACCORD 18-UNICANCER GI): A Randomized Phase III Study. J Clin Oncol. 2019;37(8):658-67.

8. Sessa C, ten Bokkel Huinink WW, du Bois A. Oxaliplatin in ovarian cancer. Ann Oncol. 1999;10(Suppl 1):55-7.

9. Suker M, Beumer BR, Sadot E, et al. FOLFIRINOX for locally advanced pancreatic cancer: a systematic review and patient-level meta-analysis. Lancet Oncol. 2016;17(6):801-10.

10. Hind D, Tappenden P, Tumur I, et al. The use of irinotecan, oxaliplatin and raltitrexed for the treatment of advanced colorectal cancer: systematic review and economic evaluation. Health Technol Assess. 2008;12(15):iii-ix, xi-162.

11. Martinez-Balibrea E, Martinez-Cardus A, Gines A, et al. Tumor-Related Molecular Mechanisms of Oxaliplatin Resistance. Mol Cancer Ther. 2015;14(8):1767-76.

12. Lykke-Andersen J, Shu MD, Steitz JA. Human Upf proteins target an mRNA for nonsense-mediated decay when bound downstream of a termination codon. Cell. 2000;103(7):1121-31.

13. Liu C, Karam R, Zhou Y, et al. The UPF1 RNA surveillance gene is commonly mutated in pancreatic adenosquamous carcinoma. Nat Med. 2014;20(6):596-8.

14. Chang L, Li C, Guo T, et al. The human RNA surveillance factor UPF1 regulates tumorigenesis by targeting Smad7 in hepatocellular carcinoma. J Exp Clin Cancer Res. 2016;35:8.

15. Zhou Y, Li Y, Wang N, et al. UPF1 inhibits the hepatocellular carcinoma progression by targeting long non-coding RNA UCA1. Sci Rep. 2019;9(1):6652.

16. Mani SA, Guo W, Liao MJ, et al. The epithelial-mesenchymal transition generates cells with properties of stem cells. Cell. 2008;133(4):704-15.

17. Zhu CC, Chen C, Xu ZQ, et al. CCR6 promotes tumor angiogenesis via the AKT/NF-kappaB/VEGF pathway in colorectal cancer. Biochim Biophys Acta Mol Basis Dis. 2018;1864(2):387-97.

18. Clarke MF, Dick JE, Dirks PB, et al. Cancer stem cells-perspectives on current status and future directions: AACR Workshop on cancer stem cells. Cancer Res. 2006;66(19):9339-44.

19. Kreso A, O'Brien CA, van Galen $P$, et al. Variable clonal repopulation dynamics influence chemotherapy response in colorectal cancer. Science. 2013;339(6119):543-8. 
20. Meacham CE, Morrison SJ. Tumour heterogeneity and cancer cell plasticity. Nature. 2013;501(7467):328-37.

21. Chaudhary K, Poirion OB, Lu L, et al. Deep Learning-Based Multi-Omics Integration Robustly Predicts Survival in Liver Cancer. Clin Cancer Res. 2018;24(6):1248-59.

22. Ho DW, Tsui YM, Sze KM, et al. Single-cell transcriptomics reveals the landscape of intra-tumoral heterogeneity and stemness-related subpopulations in liver cancer. Cancer Lett. 2019;459:176-85.

23. Zhu Y, Wang C, Becker SA, et al. miR-145 Antagonizes SNAl1-Mediated Stemness and Radiation Resistance in Colorectal Cancer. Mol Ther. 2018;26(3):744-54.

24. Marshall JL. Risk assessment in Stage II colorectal cancer. Oncology (Williston Park). 2010;24(1 Suppl 1):9-13.

25. Meyerhardt JA, Mayer RJ. Systemic therapy for colorectal cancer. N Engl J Med. 2005;352(5):47687.

26. Yu T, Guo F, Yu Y, et al. Fusobacterium nucleatum Promotes Chemoresistance to Colorectal Cancer by Modulating Autophagy. Cell. 2017;170(3):548-63. e16.

27. Diaz-Rubio E, Sastre J, Zaniboni A, et al. Oxaliplatin as single agent in previously untreated colorectal carcinoma patients: a phase II multicentric study. Ann Oncol. 1998;9(1):105-8.

28. Rothenberg ML, Oza AM, Bigelow RH, et al. Superiority of oxaliplatin and fluorouracil-leucovorin compared with either therapy alone in patients with progressive colorectal cancer after irinotecan and fluorouracil-leucovorin: interim results of a phase III trial. J Clin Oncol. 2003;21(11):2059-69.

29. Feng Q, Jagannathan S, Bradley RK. The RNA Surveillance Factor UPF1 Represses Myogenesis via Its E3 Ubiquitin Ligase Activity. Mol Cell. 2017;67(2):239-51. e6.

30. El-Bchiri J, Buhard O, Penard-Lacronique V, et al. Differential nonsense mediated decay of mutated mRNAs in mismatch repair deficient colorectal cancers. Hum Mol Genet. 2005;14(16):2435-42.

31. Bokhari A, Jonchere $V$, Lagrange A, et al. Targeting nonsense-mediated mRNA decay in colorectal cancers with microsatellite instability. Oncogenesis. 2018;7(9):70.

32. Nassar D, Blanpain C. Cancer Stem Cells: Basic Concepts and Therapeutic Implications. Annu Rev Pathol. 2016;11:47-76.

33. Chikamori K, Grabowski DR, Kinter M, et al. Phosphorylation of serine 1106 in the catalytic domain of topoisomerase II alpha regulates enzymatic activity and drug sensitivity. J Biol Chem. 2003;278(15):12696-702.

34. Grozav AG, Chikamori K, Kozuki T, et al. Casein kinase I delta/epsilon phosphorylates topoisomerase Ilalpha at serine-1106 and modulates DNA cleavage activity. Nucleic Acids Res. 2009;37(2):382-92.

35. Labbe DP, Sweeney CJ, Brown M, et al. TOP2A and EZH2 Provide Early Detection of an Aggressive Prostate Cancer Subgroup. Clin Cancer Res. 2017;23(22):7072-83.

36. Pei YF, Yin XM, Liu XQ. TOP2A induces malignant character of pancreatic cancer through activating beta-catenin signaling pathway. Biochim Biophys Acta Mol Basis Dis. 2018;1864(1):197-207. 
37. Zeng S, Liu A, Dai L, et al. Prognostic value of TOP2A in bladder urothelial carcinoma and potential molecular mechanisms. BMC Cancer. 2019;19(1):604.

38. Zhang $\mathrm{R}, \mathrm{Xu} \mathrm{J}$, Zhao J, et al. Proliferation and invasion of colon cancer cells are suppressed by knockdown of TOP2A. J Cell Biochem. 2018;119(9):7256-63.

39. Delgado JL, Hsieh CM, Chan NL, et al. Topoisomerases as anticancer targets. Biochem J. 2018;475(2):373-98.

40. Meisenberg C, Gilbert DC, Chalmers A, et al. Clinical and cellular roles for TDP1 and TOP1 in modulating colorectal cancer response to irinotecan. Mol Cancer Ther. 2015;14(2):575-85.

41. Kim HJ, Moon SJ, Kim SH, et al. DBC1 regulates Wnt/beta-catenin-mediated expression of MACC1, a key regulator of cancer progression, in colon cancer. Cell Death Dis. 2018;9(8):831.

42. Dimberg J, Skarstedt M, Slind Olsen R, et al. Gene polymorphism in DNA repair genes XRCC1 and XRCC6 and association with colorectal cancer in Swedish patients. APMIS. 2016;124(9):736-40.

\section{Figures}



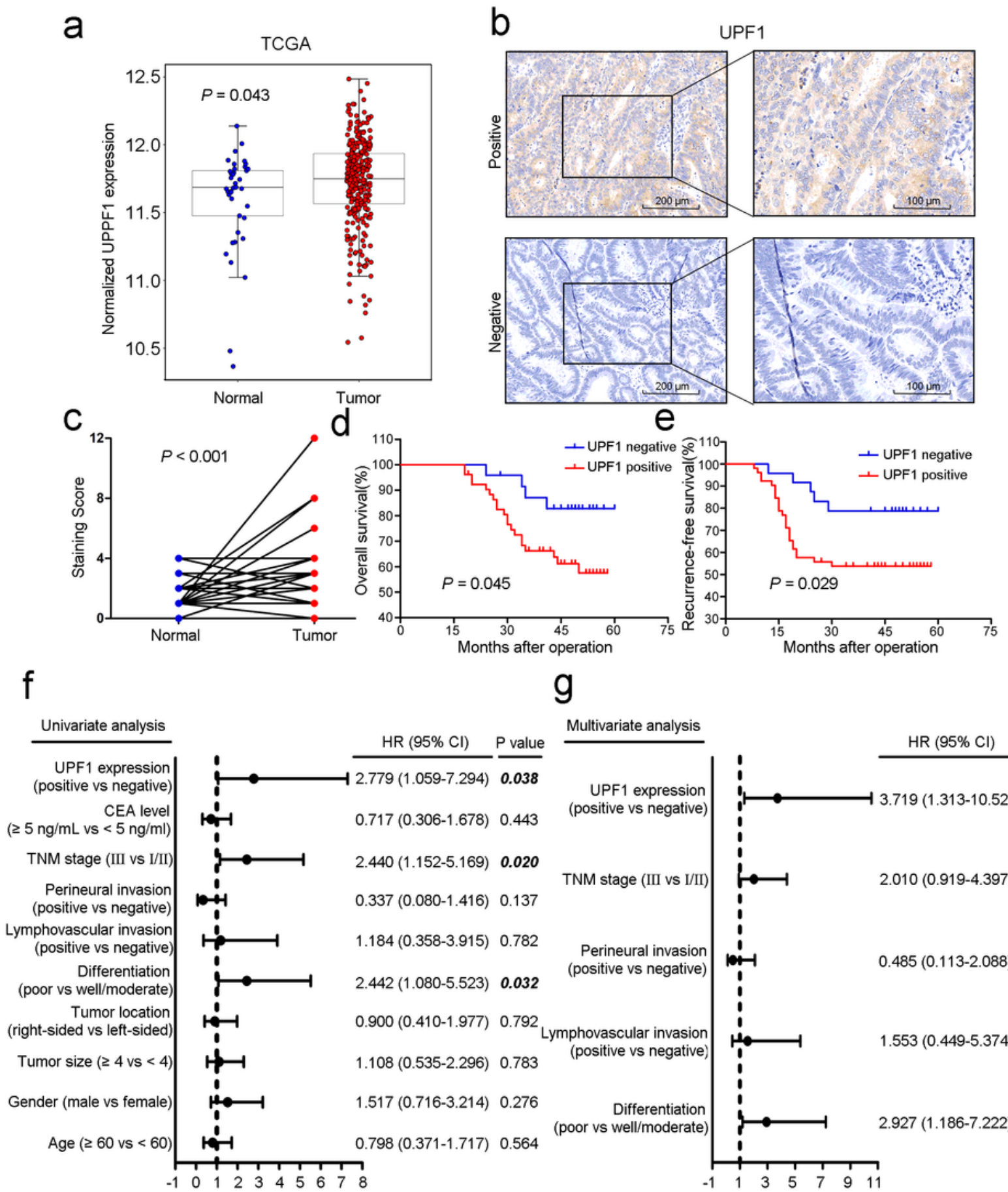

\section{g}

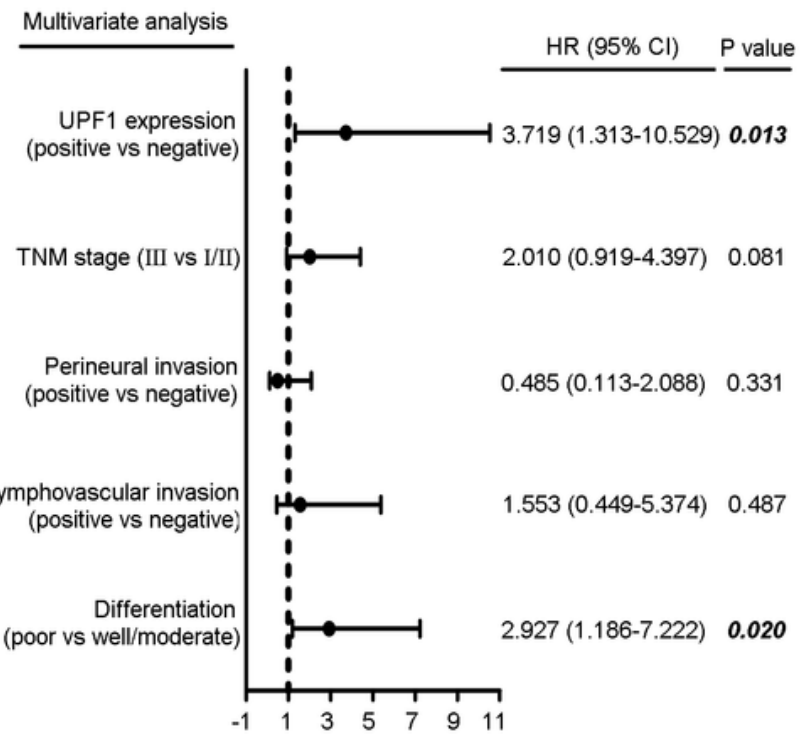

\section{Figure 1}

UPF1 is upregulated in CRC and predicted a poor prognosis. (a) UPF1 was overexpressed in CRC tissues in mRNA level from TCGA datasets. (b) Images of UPF1 in IHC staining in CRC tissue microarray, scale bar, $200 \mu \mathrm{m}$ and $100 \mu \mathrm{m}$. (c) UPF1 was overexpressed in CRC tissues in protein levels by IHC staining in TMA. (d-e) UPF1 positive predicted worse OS and RFS in CRC patients. (f-g) Univariate and multivariate 
COX regression analysis revealed that UPF1 was an independent risk factor for recurrence in CRC patients.

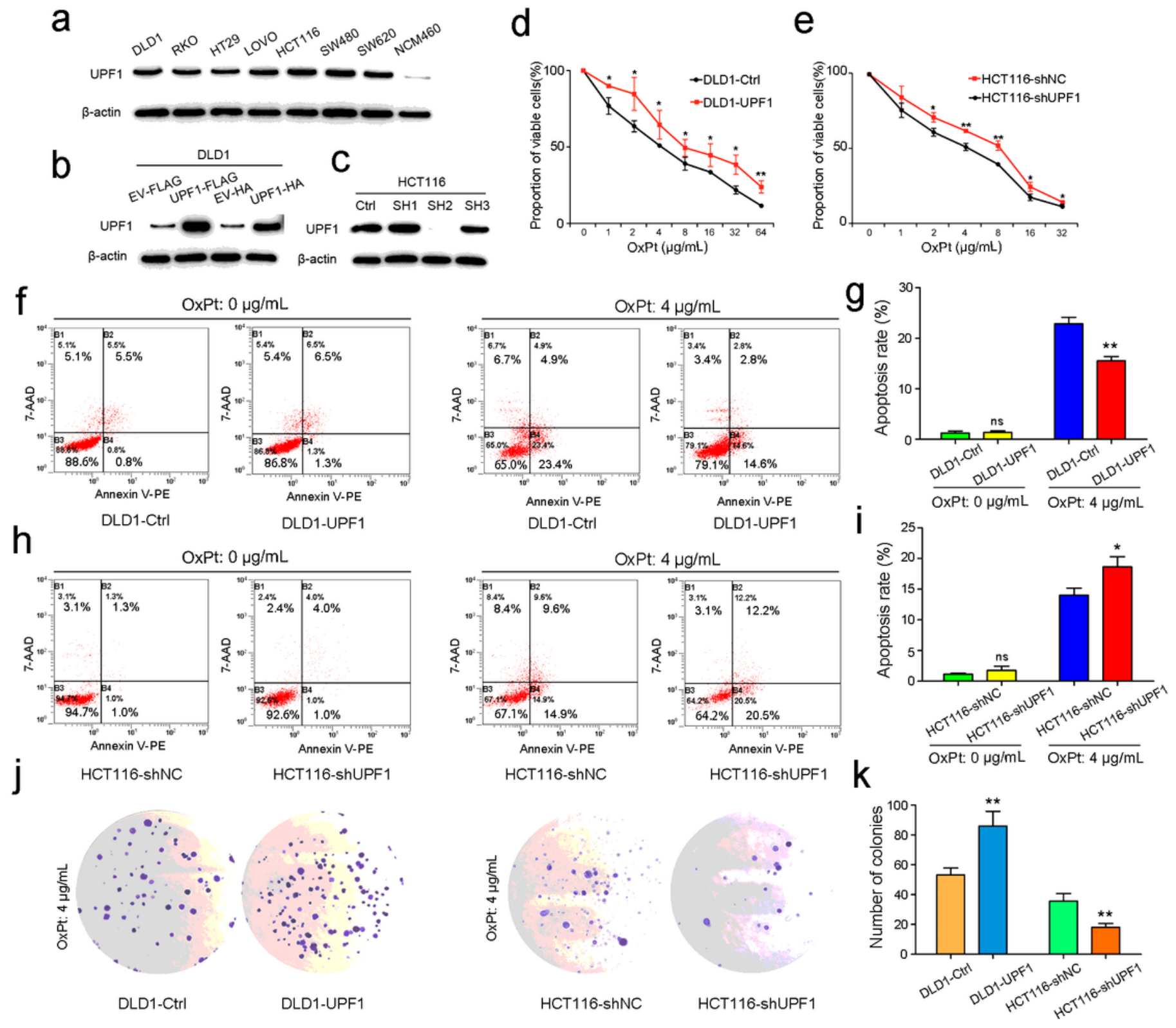

Figure 2

UPF1 induced chemoresistance to oxaliplatin (OxPt) in CRC in vitro. (a) The baseline expression of UPF1 in CRC cell lines and normal colonic epithelial cell line detected by western blot. (b-c) Overexpression and knockdown of UPF1 in DLD1 and HCT116 respectively. (d-e) Cell viability of stable CRC cell lines treated with a gradient concentration of oxaliplatin assessed by CCK-8 assay. $(\mathrm{f}-\mathrm{i})$ Apoptosis rate of stable CRC cell lines treated with or without oxaliplatin assessed by flow cytometry. (j-k) Clone formation of stable $\mathrm{CRC}$ cell lines treated with oxaliplatin. 

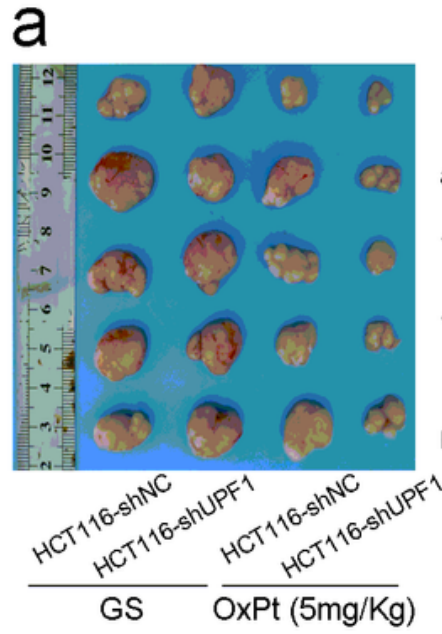

b

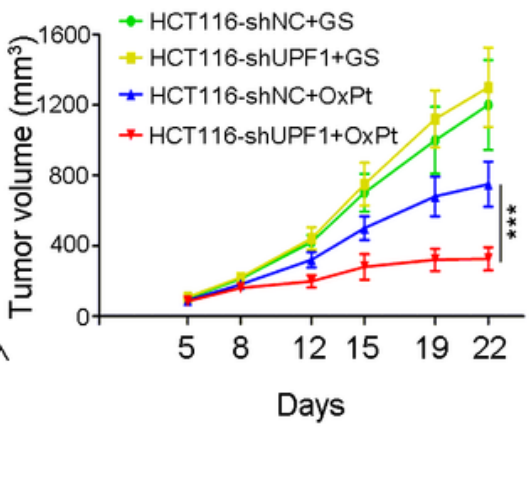

C

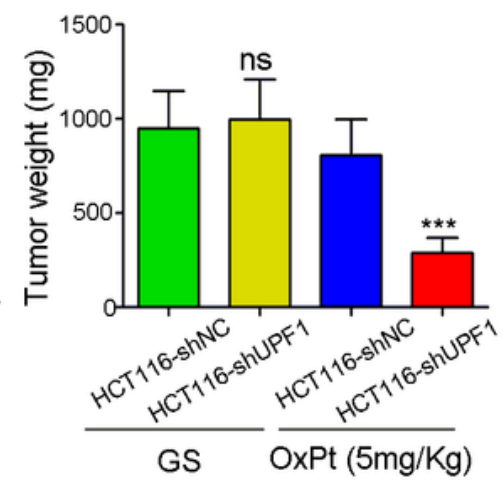

d

HCT116-shNC+GS HCT116-shUPF1+GS HCT116-shNC+OxPtHCT116-shUPF1+OxPt
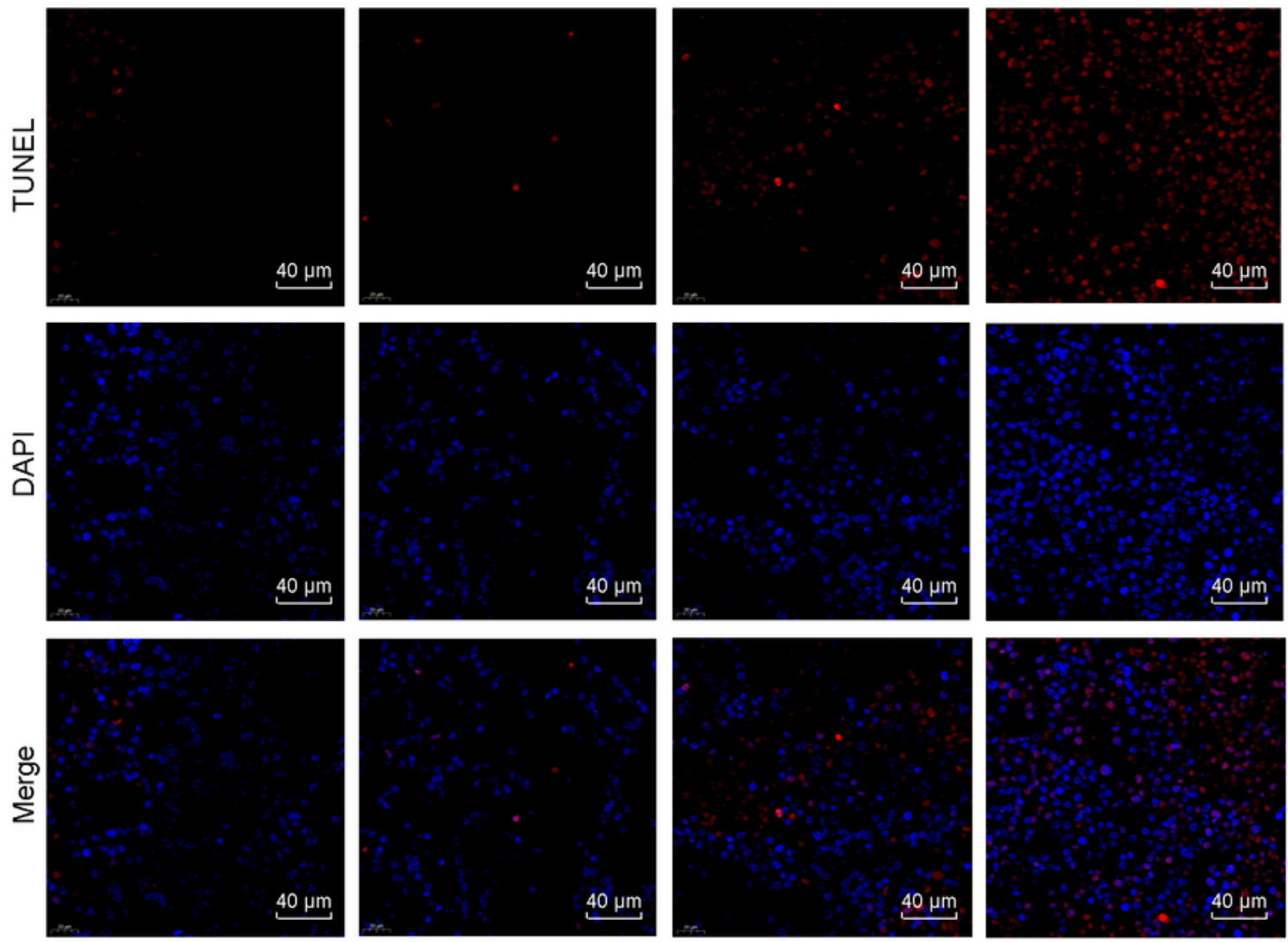

\section{Figure 3}

UPF1 promoted chemoresistance to oxaliplatin in CRC in vivo. (a) xenografts of HCT116-shNC and HCT116-shUPF1 treated with or without oxaliplatin. (b) Tumor volume in nude mice bearing HCT116 cells in different groups. Tumor volume equaled length $\times$ width^ $2 \times 0.5$ and was measured twice a week. (c) Tumor weight in nude mice bearing HCT116 cells in different groups. (d) TUNEL assay was performed to detect tumor cell apoptosis in xenograft tumor tissues, scale bar, $40 \mu \mathrm{m}$. 


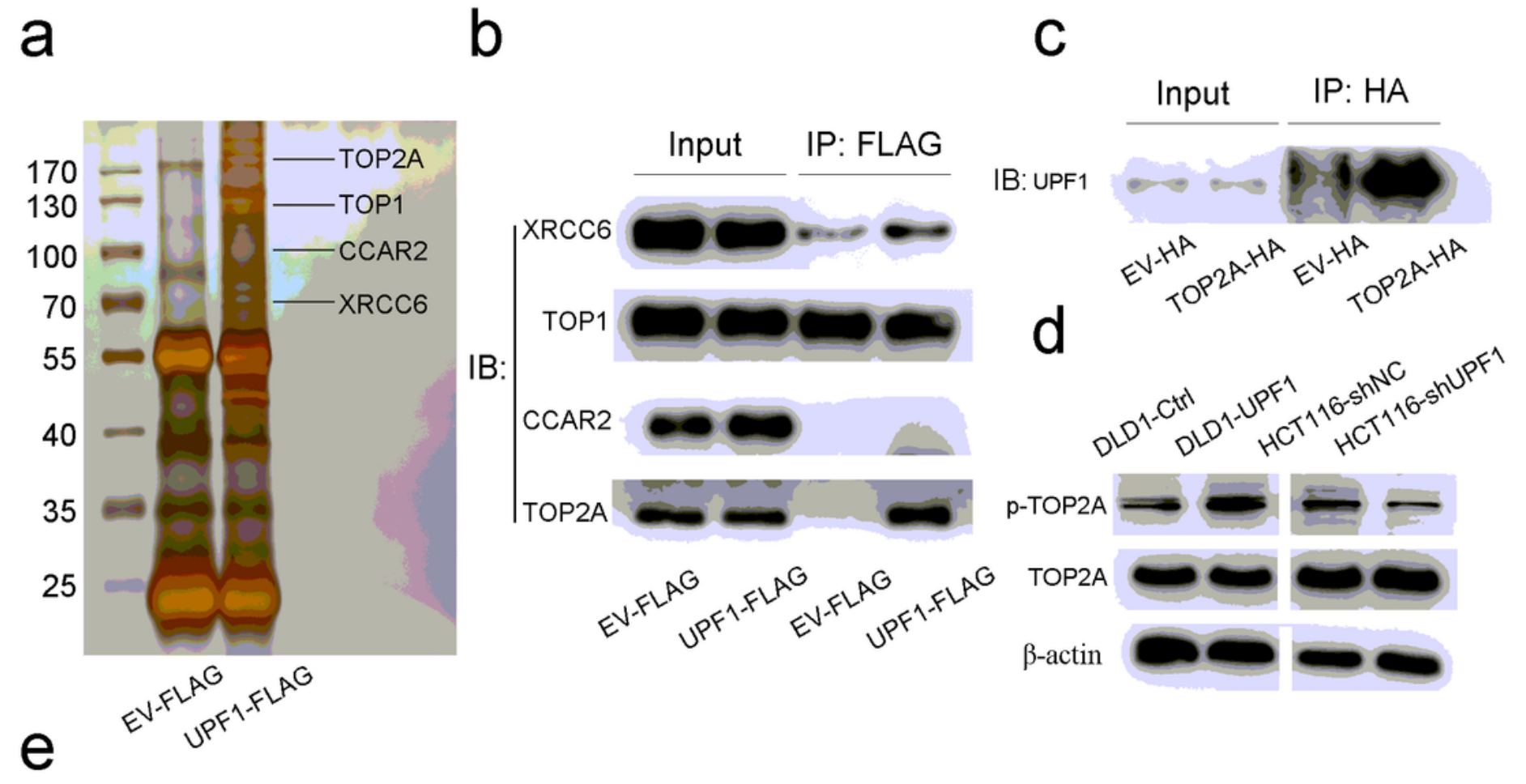

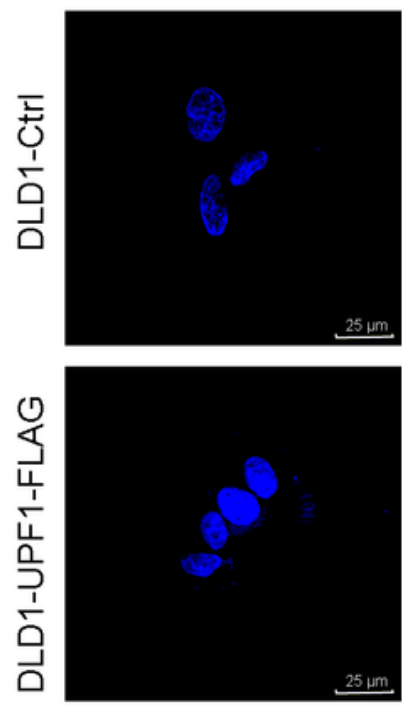

DAPI
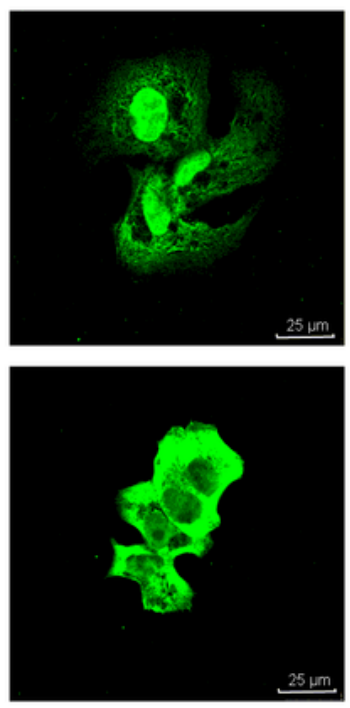

FLAG
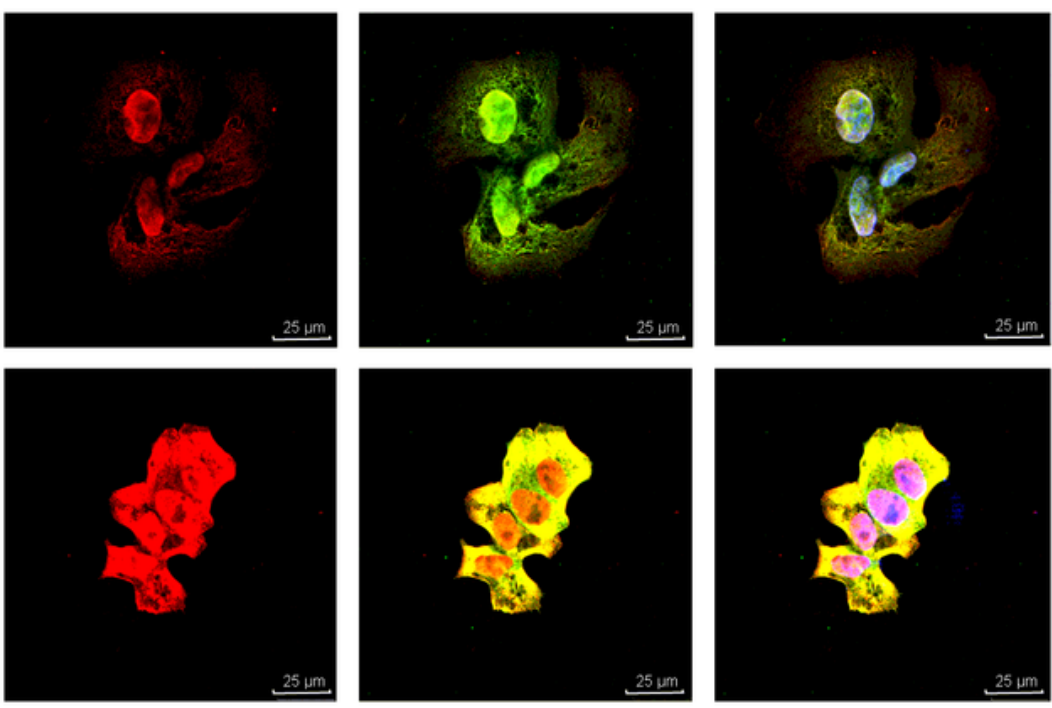

TOP2A

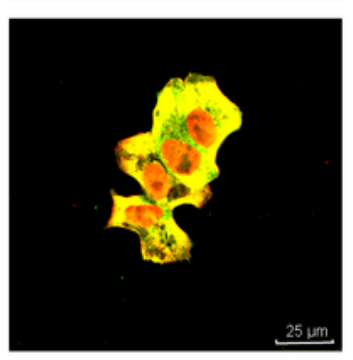

Merge1

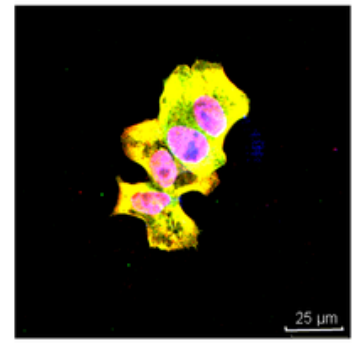

Merge2

\section{Figure 4}

UPF1 interacted with TOP2A and increased phosphorylated TOP2A. (a) signature bands of DLD1-UPF1 cell line emerged after silver staining. The location of TOP2A, TOP1, CCAR2 and XRCC6 were marked. (bc) Interaction of UPF1 and TOP2A proved in co-IP assay. (d) UPF1 had no influence on the total expression of TOP2A but raised phosphorylated TOP2A at the site of Ser1106. (e) Images of ICC assay showed interaction between UPF1 and TOP2A, scale bar, $25 \mu \mathrm{m}$. 


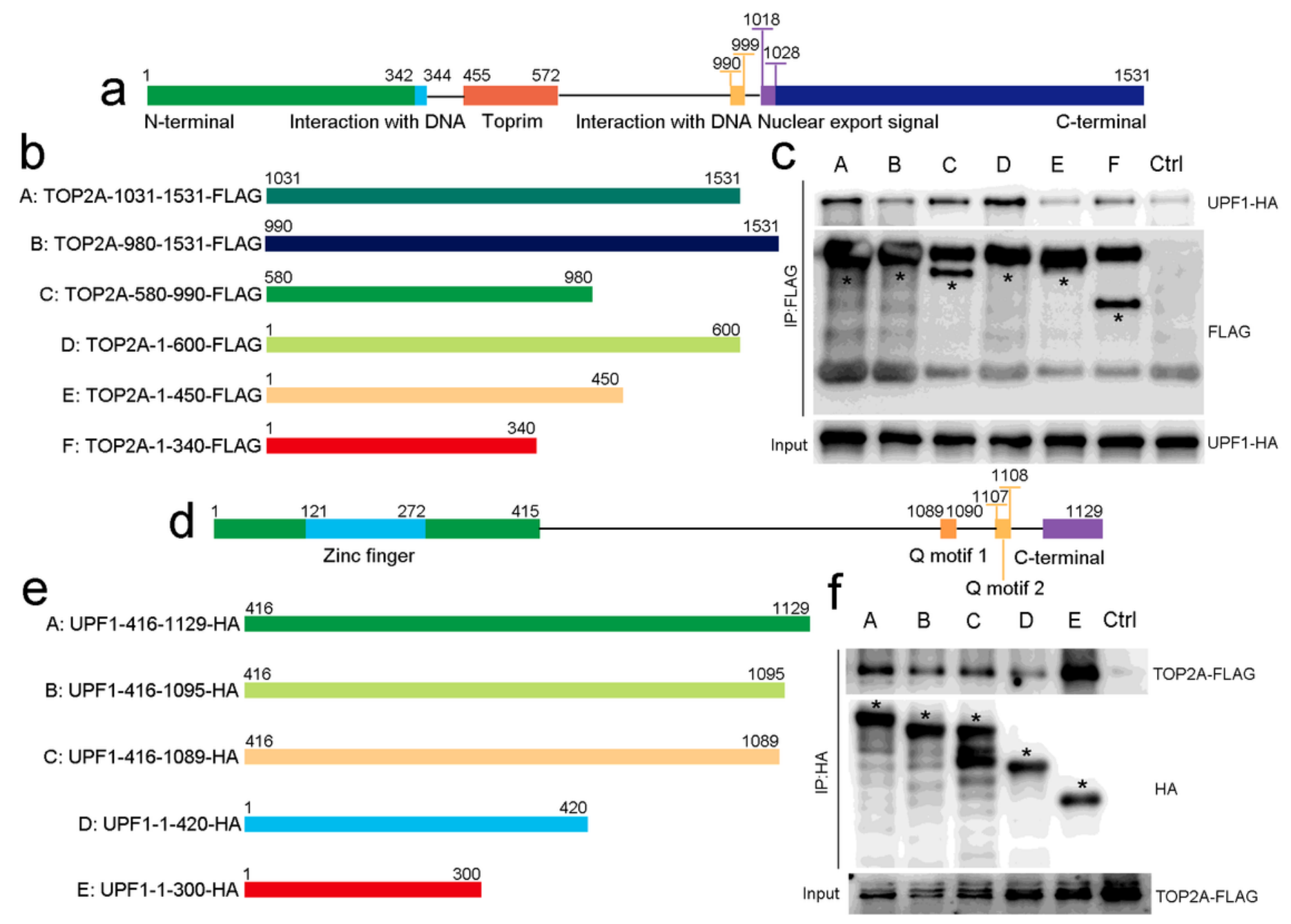

\section{Figure 5}

Co-IP assays using truncated mutants identified the precise binding region between TOP2A and UPF1. (a) Schematic diagram of key domains in TOP2A. (b) Six truncated mutants of TOP2A fused with FLAG tag were generated according to its key domains. (c) Co-IP assays were performed of the lysates from HEK293T cells transfected with HA-tagged UPF1and FLAG-tagged truncated mutants of TOP2A. (d) Schematic diagram of key domains in UPF1. (e) Five truncated mutants of UPF1 fused with HA tag were generated according to its key domains. (f) Co-IP assays were performed of the lysates from HEK-293T cells transfected with FLAG-tagged TOP2A and HA-tagged truncated mutants of UPF1. 


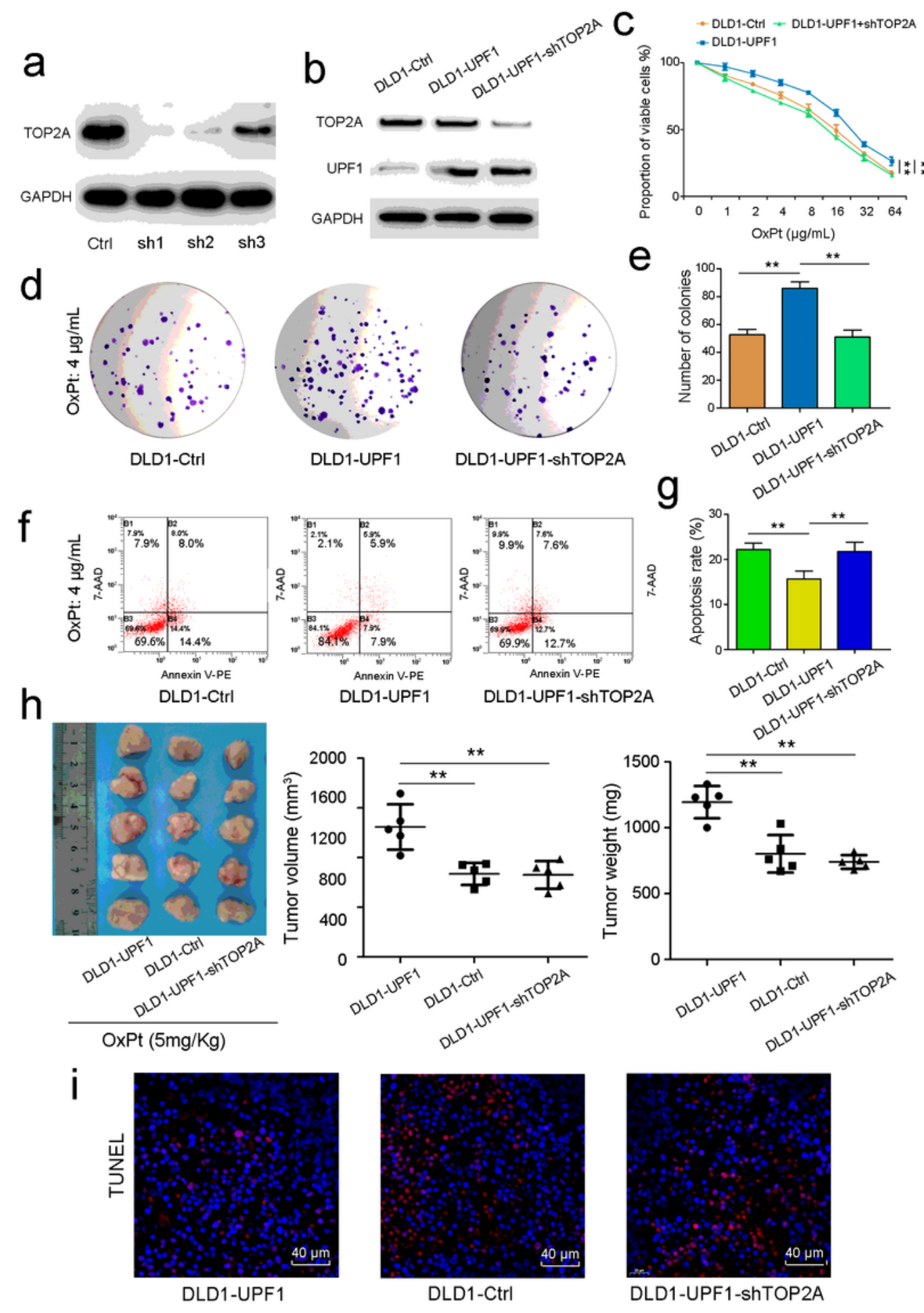

\section{Figure 6}

UPF1-induced oxaliplatin resistance was abolished by silencing the TOP2A gene in vitro and in vivo. (a-b) TOP2A was silenced using small hairpin RNAs. (c) Drug cytotoxicity assay, (d, e) clone formation assay and $(f, g)$ flow cytometry showed abrogation of chemoresistance to oxaliplatin induced by UPF1 by knockdown of TOP2A. (h) xenografts of DLD1-UPF1, DLD1-Ctrl and DLD1-UPF1-shTOP2A treated with 
oxaliplatin and tumor volume and weight of xenografts in different groups. (i) TUNEL assay was performed to detect tumor cell apoptosis in xenograft tumor tissues, scale bar, $40 \mu \mathrm{m}$.

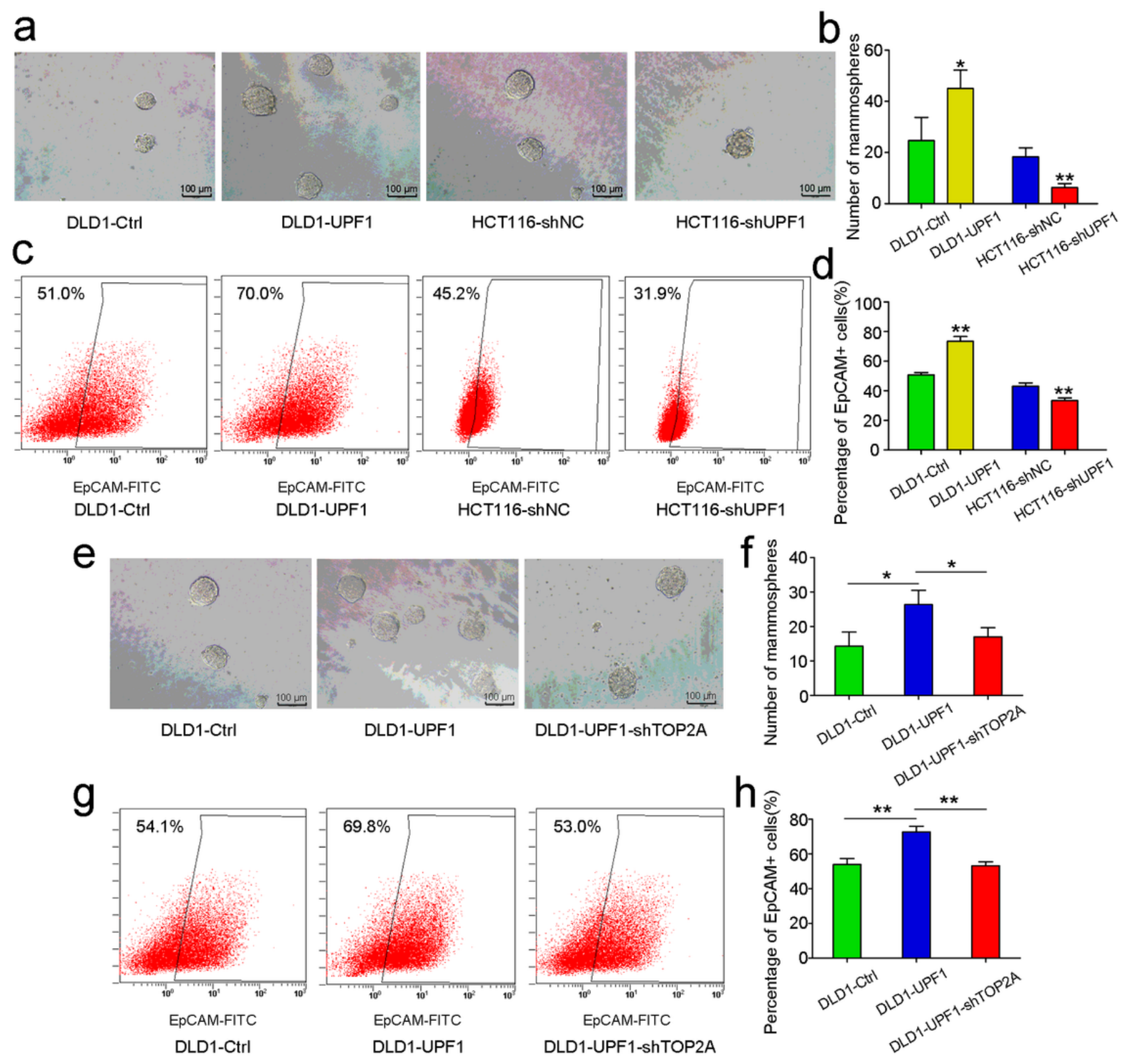

Figure 7

UPF1 maintains stemness of CRC cell lines. (a-b) The ability of mammosphere formation was enhanced after upregulation of UPF1 in DLD1 and was attenuated after knockdown of UPF1 in HCT116, scale bar, $100 \mu \mathrm{m}$. (c-d) The percentage of EpCAM-positive cells was raised in DLD1-UPF1 and diminished in HCT116-shUPF1 compared with control. (e-f) Silencing TOP2A abrogated enhancement of the ability of 
mammosphere formation induced by UPF1, scale bar, $100 \mu \mathrm{m}$. (g-h) Silencing TOP2A down regulated the rise of the percentage of EpCAM-positive cells induced by UPF1.

\section{Supplementary Files}

This is a list of supplementary files associated with this preprint. Click to download.

- FigureS1.tif

- FigureS2.tif

- Tables1.docx

- TableS2.docx

- TableS3.xlsx 